\title{
FURTHER MODEL STUDY OF THE RADIATION OF ELASTIC WAVES FROM A DIPOLE SOURCE
}

\author{
By John H. Healy and Frank Press
}

\section{INTRODUCTION}

Earthquake-Generated compressional and shear waves have been used extensively in recent years for studying the mechanism at the focus. ${ }^{1}$ In general the procedure is to compare the radiation pattern of these waves with the theoretical pattern derived from simple sources such as singlets, dipoles, quadrupoles, etc. The importance of these investigations to our understanding of tectonic processes makes it desirable to examine the methods with a view toward placing them on a firm theoretical and experimental foundation.

One approach is to use the methods of ultrasonic-model seismology to determine whether or not the radiation pattern postulated for certain sources is actually observed. The effects of various perturbations such as finite fault length and elastic heterogeneity near the focus can be conveniently examined.

\section{Resulits of Previous Work}

Two-dimensional ultrasonic-model studies of the radiation pattern from dipole sources have been described elsewhere. ${ }^{2}$ The boundary conditions which obtain at a fault were approximately allowed for by placing a grease-filled or glue-filled slit between the elements of the dipole. We do not pretend that this is a true model of a fault; however, it may represent a closer approximation than a simple dipole. The results showed that the quadrant distribution of alternate compressions and rarefactions occurs for compressional waves from dipole sources with and without slits. The shear-wave distribution is significantly altered, however, when the slit is present (fig. 1). Instead of a node at $0^{\circ}$, anomalous shear waves with sizable amplitudes are observed, with nodes occurring at about $\pm 30^{\circ}$. This occurs whether or not the slit is greased or glued. It was concluded on the basis of this work that faultmechanism studies using compressional waves had a reasonable basis but that additional work was needed before shear-wave data could be properly interpreted.

\section{New REsults}

In this paper we demonstrate one possible mechanism for the origin of the anomalous shear waves. A two-dimensional study of elastic waves generated by a singlet source adjacent to a long slit reveals that Rayleigh waves are excited. These waves

Manuscript received for publication October 7, 1958.

The preparation of this paper was sponsored by the Office of Ordnance Research, U. S. Army. California Institute of Technology, Division of Geological Sciences, contribution no. 900 .

1 Perry Byerly, "Nature of Faulting as Deduced from Seismograms," Geol. Soc. Am., Spec. Pap. 62: 74-86 (1955); V. I. Kelis-Borok, "Methods and Results of the Investigation of the Earthquake Mechanism," Trav. Sci. Assoc. Seism. U.G.G.I., 19: 383-394 (1956).

${ }^{2}$ Frank Press, "Elastic Wave Radiation from Faults in Ultrasonic Models," Trav. Sci. Assoc. Seism. U.G.G.I. (in press); Y. Kato and A. Takagi, "Seismic Studies: Part 3," Sci. Rep. Toboku Univ., 5th ser., 8: 212-224 (1957). 

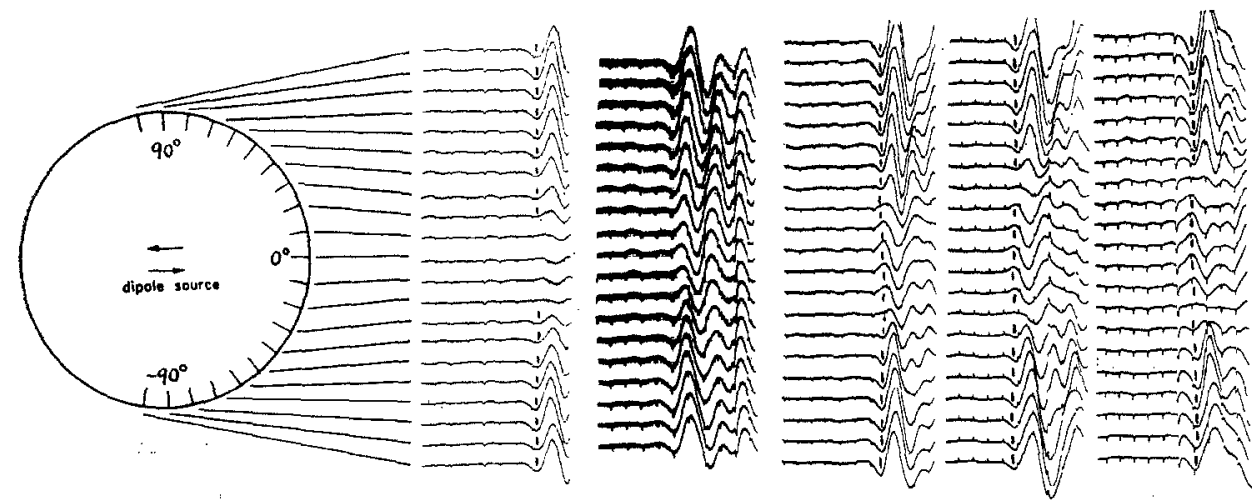

Fig. 1. Shear waves from a dipole source: A, no slit; B, 0.4-inch slit; C, 0.8-inch slit; D, 1.6-inch slit; E, 1.6-inch slit glued. Downward trace motion corresponds to counterclockwise rotational motion on the model.

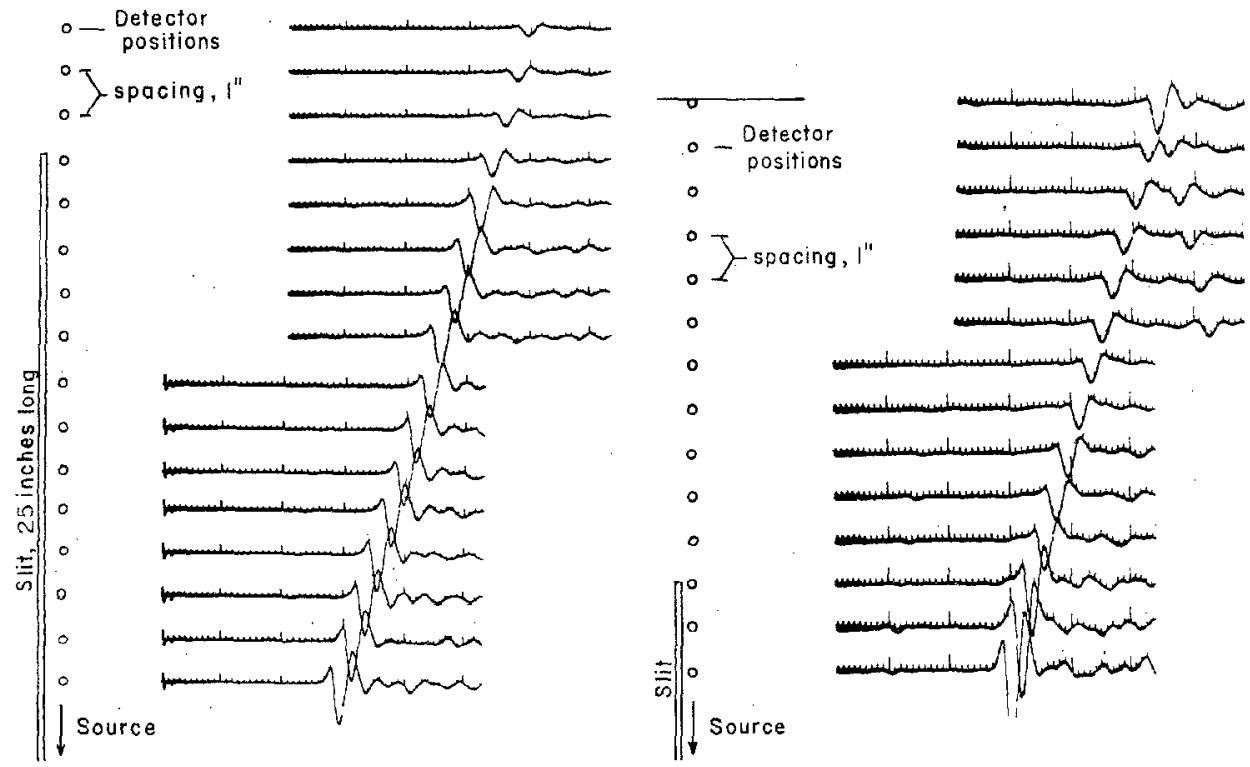

Fig. 2 (left). Seismograms from transverse detectors illustrating conversion of Rayleigh waves to shear waves. Singlet source located adjacent to slit 25 inches from end; impulse acts in direction of slit. Time-mark interval is 10 microseconds.

Fig. 3 (right). Continuation of figure 2. 


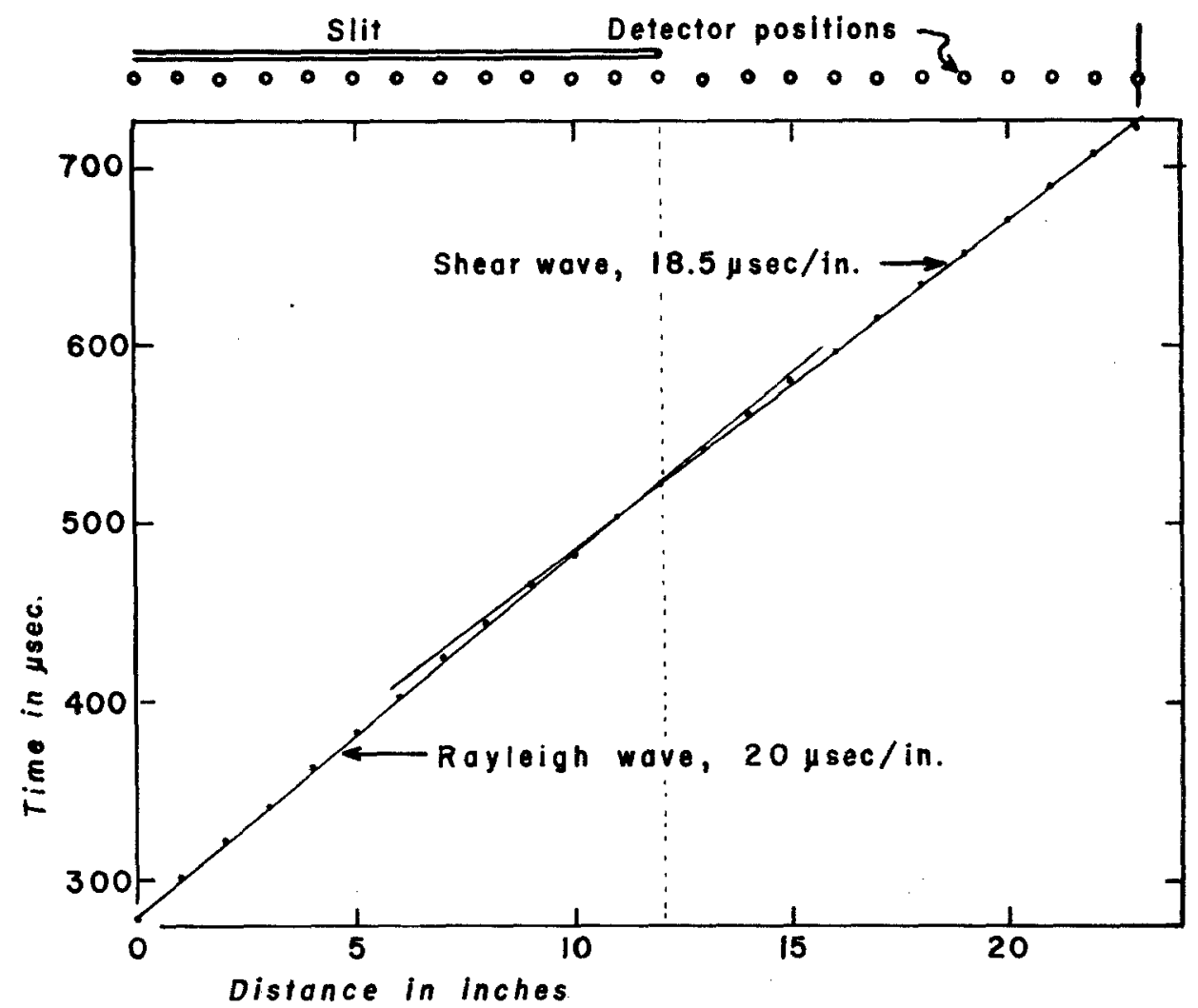

Fig. 4. Travel-time curves showing break in velocity associated with Rayleigh to shear conversion.

travel along the edge to the end of the slit, from which point they are transmitted as shear waves into the body of the medium. A reflected Rayleigh wave also occurs.

Figures 2 and 3 show the actual seismograms obtained at various positions along the slit. The source is 25 inches from the edge of the slit. Rayleigh waves occur with their characteristic nondispersed pulse shape, and the converted shear waves maintain this pulse shape with diminished amplitude. The direction of initial motion in the converted shear wave agrees with that reported earlier for the anomalous shear waves and is contrary to the initial motion of the direct shear waves. It also follows from the theory of Rayleigh waves that the second element of the dipole acting on the other side of the slit would excite converted shear waves having the same sense, thereby further increasing the amplitudes of anomalous shear waves.

Figure 4 shows the travel-time curves for the Rayleigh and shear waves, the indicated reciprocal velocities being in the proper ratio.

The two-dimensional Rayleigh wave diminishes in amplitude with distance as a result of absorption only. At large distances from the edge the shear wave should fall off with distance, owing to cylindrical divergence and a smaller absorption. ${ }^{3}$

\footnotetext{
${ }^{3}$ Frank Press and John Healy, "Absorption of Rayleigh Waves in Low-Loss Media," Jour. Appl. Phys., 28: 1323-1325 (1957).
} 
Detector positions

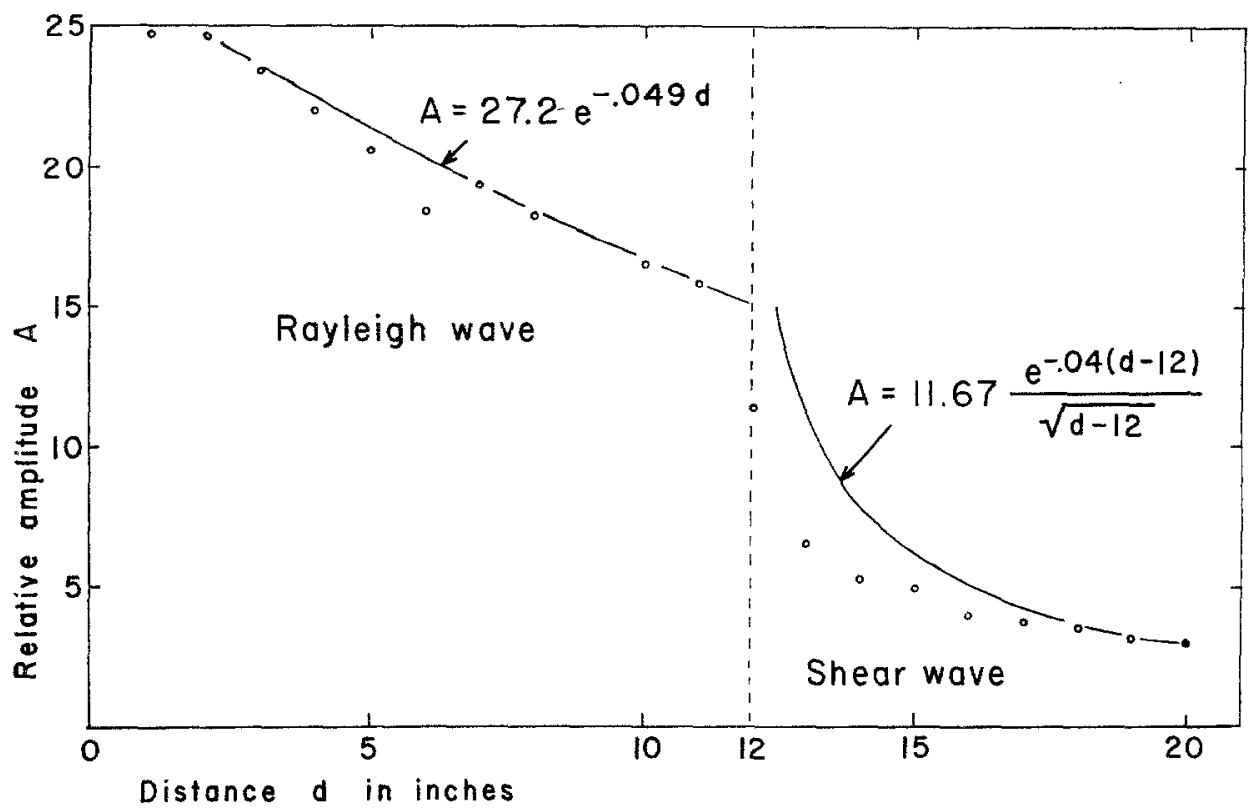

Fig. 5. Experimental Rayleigh-wave and shear-wave amplitude data.
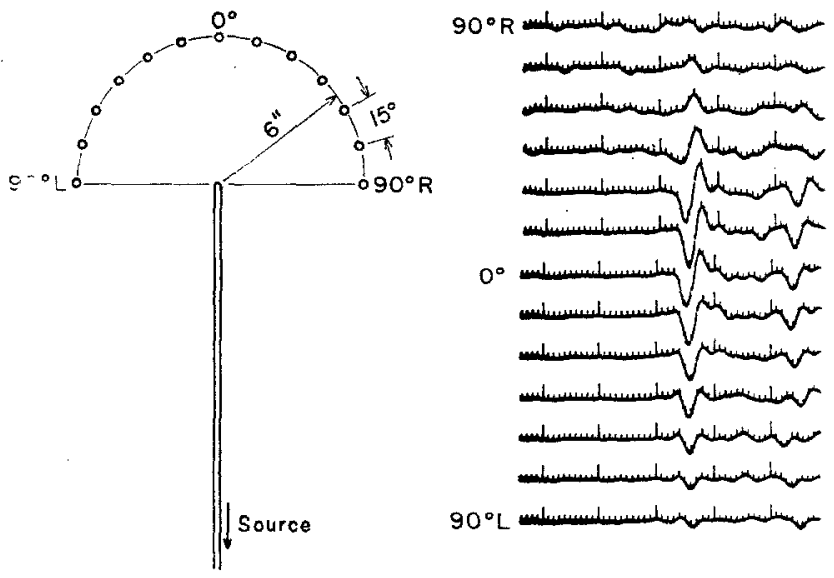

Fig. 6. Shear waves observed at various azimuths from secondary source. 


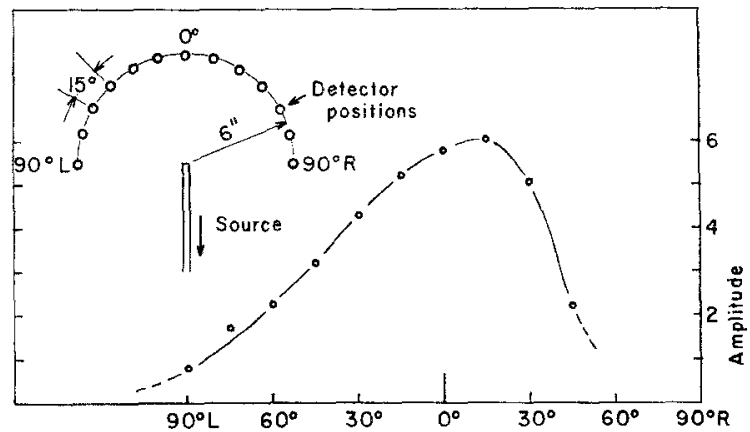

Fig. 7. Amplitude variation of shear waves with azimuth from secondary"source.

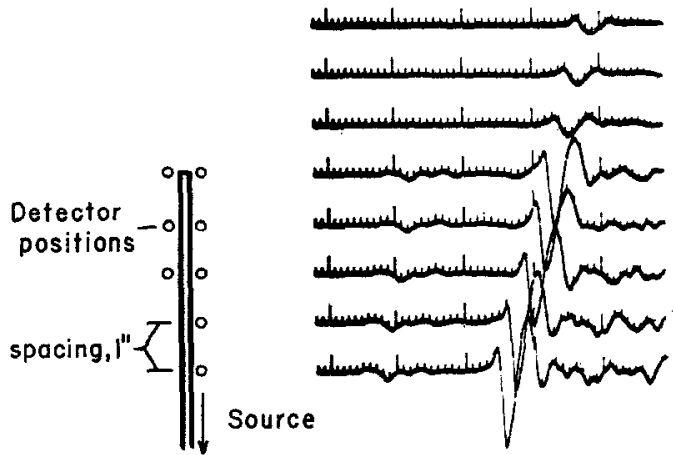

Fig. 8. Reflected Rayleigh waves. Top three traces correspond to detector positions on left side of slit.

Experimental amplitude data are presented in figure 5. The curves are drawn for comparison purposes only, to fit the Rayleigh-wave amplitudes at all positions and the shear-wave amplitudes only at the large distances. It is not expected that the simple law of cylindrical divergence would fit the shear-wave data near their secondary source.

The variation of shear-wave amplitude with azimuth from the secondary source is of interest because of its potential utility in dynamic interpretation methods. The seismograms presented in figure 6 were obtained along a semicircle centered on this source. There is a variation in pulse shape as well as amplitude with position. The amplitude data in figure 7 show a surprising feature, namely, that the decrease in amplitude with increasing azimuth is more rapid on the side adjacent to the source. The conversion from Rayleigh to compressional waves was not observed.

In figure 8 the phenomenon of reflected Rayleigh waves is shown. It is seen that the reflected Rayleigh waves occur primarily on the other side of the slit for the case where the slit is not glued. 


\section{Discussion}

It can easily be shown that the conditions of tangential slipping and continuity in normal stress and displacement which obtain at an idealized fault plane are sufficient for the propagation of Rayleigh waves. Although more difficult to demonstrate, it is not unreasonable to expect that the zone of reduced rigidity adjacent to faults would favor the propagation of interface waves even if tangential slipping is not fully realizable. The experimental data, therefore, show one mechanism for the generation of anomalous shear waves involving conversion from Rayleigh waves to shear waves at the end of a fault. It is difficult to state whether or not this effect can occur as a natural phenomenon, in view of the uncertainty of the earthquake mechanism. However, the fact that faulting proceeds with a finite velocity over a finite distance suggests very strongly that a secondary source of shear waves may occur at the end of the fault in a manner analogous to that of the experimental results here reported. That Rayleigh waves associated with the fault interface are indeed pertinent is suggested by the work of Yoffe ${ }^{4}$ in which it is shown that a system of surface waves can be found which satisfies the boundary conditions for a moving Griffith crack. It is concluded that anomalous shear waves may occur in nature, in which case proper allowance for them must be made in studies of the mechanism of the focus.

\footnotetext{
${ }^{4}$ E. H. Yoffe, "The Moving Griffith Crack," Phil. Mag., 42: 739-750 (1951).
} 